\title{
Case - Repair of extensive testicular rupture: A unique use for biosynthetic grafts
}

Paul Nhu Doan; Jim Yip; Henry Wang; Daniel Chia, Lawrence H.C. Kim; Audrey Wang Westmead Hospital, Sydney, Australia

Cite as: Can Urol Assoc J 2019 June 17; Epub ahead of print. http://dx.doi.org/10.5489/cuaj.6052

Published online June 17, 2019

$* * *$

\section{Introduction}

Testicular rupture is defined as the disruption of the tunica albuginea of the testes. It may occur as a result of blunt or penetrating trauma. ${ }^{1}$ In cases of testicular rupture, urgent surgical management is required for effective care of the patient and involves primary closure of the defect. $^{2}$ However, if the testicular rupture is extensive and primary closure is not possible, the patient must either undergo orchiectomy or an alternative method must be used to close the defect. In this case series, we present three cases of extensive testicular rupture, one penetrating and two blunt, where a biosynthetic graft was used to successfully close the defect and preserve the testes.

\section{Case 1}

A 21-year-old man presented to the Emergency Department brought in by ambulance with multiple gunshot wounds to his scrotum and both thighs. Primary survey showed stable vital signs. A secondary survey revealed two penetrating entry and exit wounds in his scrotum (one on the left side and one on the right). It also revealed three entry wounds on his thighs (two on the right medial thigh and one on the left medial thigh) with two corresponding exit wounds on the posterior side in his right buttock and right thigh. The penis was uninjured. CT scan of the pelvis revealed a swollen scrotum and active contrast blushing in right side of the scrotum. Haemoglobin was stable at 113.

Scrotal exploration was performed which revealed severe right testicular injury and rupture. A significantly contaminated area was debrided to healthy tissues and repair of a large defect in the tunica albuginea was achieved with a biosynthetic graft (Biodesign Surgisis) to the remaining tunica edges. The graft was secured with 4'0 PDS sutures. A Penrose drain left in the scrotum.

Postoperative management included admission into a High Dependency Unit with intravenous Cephazolin for 24 hours, intravenous fluids and scrotal support dressings. The patient was discharged with seven days of oral Cephalexin. Ultrasound was performed seven days after discharge and showed perfusion in both testes. Follow up ultrasound of the scrotum in one month also showed testicular blood flow bilaterally. 


\section{Case 2}

A 25-year-old man who presented with severe right testicular pain after he sustained blunt trauma to the right scrotum by a cricket ball. The right scrotum was extremely tender and swollen and the preoperative ultrasound showed significant haematocele with a disruption of tunica albuginea in keeping with a ruptured testis.

The scrotum was explored and revealed extensive rupture of the right testis. Careful debridement was performed until healthy seminiferous tubules were demonstrated. The defect coverage was achieved with a biosynthetic graft (Biodesign Surgisis). He was discharged well the next day following 24 hours of IV cephazolin and 1 week of oral cephalexin. Follow-up 1-week and 4-week postoperative ultrasounds showed a well perfused right testis.

\section{Case 3}

A 28-year-old male presented to Westmead Hospital for a 1-day history of right testicular pain and swelling following a collision with a cricket ball.

On examination the right testis was grossly swollen and tender. Ultrasound showed large areas of haematoma and an ill-defined tunica albuginea suspicious of testicular rupture. The patient underwent scrotal exploration. The right testis had a global dusky appearance. Rupture was seen at the inferior pole with extruded seminiferous tubules and haematoma on view. (Figure 1) Judicious debridement was performed with approximately 30\% of right testis volume lost, the remaining defect was difficult to close primarily. (Figure 2) There was a noticeable return of colour of the testis with warm packs. The decision was made to close the defect with a biosynthetic graft (Biodesign Surgisis). (Figure 3) The patient was discharged home following 24 hours of IV cephazolin on 1 week of cephalexin, analgesia and scrotal support.

On follow-up the patient reported good recovery with repeat U/S at 1 week showing haematoma but normal vascularity of the right testis and repeat U/S at 6 weeks showing resolving haematoma and a testis volume of $7.5 \mathrm{cc}$ as expected. On examination the testes appeared normal with no swelling, tenderness or signs of infection.

\section{Discussion}

Extensive injury of the testes is rare secondary to the anatomical location of the testes between the thighs and their mobile nature within the scrotal sac. ${ }^{3}$ Treatment of testicular rupture can involve a number of different strategies. Conservative management has been reported in previous studies, but its use is controversial due to the increased risk of infection, atrophy and impaired fertility caused by haematocele and necrotic tissue. ${ }^{4}$ The current recommendation for management of testicular rupture is early surgical intervention with scrotal exploration within 72 hours of injury. ${ }^{5}$ This has been shown to decrease rates of orchiectomy, improve testicular hormone function and improve symptom control. ${ }^{6}$

Repair of testicular rupture involves scrotal exploration with the debridement of necrotic tissue, saline irrigation and primary closure of the tunica albuginea with absorbable sutures. ${ }^{2}$ However in extensive defects, closure with the tunica albuginea may not be 
possible. In these injuries the options are orchiectomy or repair of the defect using alternative methods. In this case series we present three cases which had extensive defects of the tunica albuginea which could not be repaired with primary closure. Instead of orchiectomy we opted for the use of a biosynthetic graft to cover the defect. This technique was successfully used in each of the three cases and the patients recovered without complications. Avoiding orchiectomy allowed for the maximal preservation of testicular tissue and hormonal function.

Alternative methods for repair of extensive testicular rupture have been reported in literature and most commonly an autologous graft from the tunica vaginalis has been harvested from the ipsilateral hemiscrotum and sewn to edges of the tunica albuginea. ${ }^{7,8}$ However current literature is somewhat limited and there is not enough data to support this method as the most effective for repair of extensive testicular rupture. As well tunica vaginalis graft would not be a viable option in cases where the quality of tunica vaginalis was also compromised such as in our first case. The use of polypropylene mesh as a graft material has also been described and has been shown to preserve more testicular volume than primary closure in blunt testicular rupture. ${ }^{9}$ The use of synthetic grafts to repair rupture from gunshot wounds has been previously associated with high infection rates. ${ }^{7}$ However the infection rate may be lowered via the use of a biosynthetic graft and antibiotic prophylaxis as seen in the cases we have presented. The protection against infection with biosynthetic grafts for testicular rupture has been similarly described in the use of bovine pericardium, cadaveric pericardium and cadaveric dermis. ${ }^{10}$

We acknowledge the weaknesses of our series given the short duration of follow-up. Further studies are needed to determine the repairs effect on long-term testicular preservation and its relation to hormonal and reproductive function to be able to completely determine benefit.

\section{Conclusions}

In cases of extensive testicular rupture where primary closure is not possible, we have demonstrated that biosynthetic grafts could be a safe and feasible alternative to orchiectomy. This would facilitate the maximal testicular preservation which would potentially reduce any loss of important testicular function. 


\section{References}

1. Papoutsoglou N and Thiruchelvam N. Diagnosis and Management of Testicular Injuries. Med Surg Urol 2013. 2:2 DOI: 10.4172/2168-9857.1000108

2. Lucky M, Brown G, Dorkin T, Pearcy R, Shabbir M, et al. British Association of Urological Surgeons (BAUS) consensus document for the management of male genital emergencies - testicular trauma. BJUI 2018.121(6):840-4

3. Deurdulian C., Mittelstaedt C. A., Chong W. K., Fielding J. R. US of acute scrotal trauma: Optimal technique, imaging findings, and management. RadioGraphics 2007;27(2):357-69. doi: 10.1148/rg.272065117.

4. J. Cubillos, E.F. Reda, J. Gitlin, Zelkovic P, Palmer L. A conservative approach to testicular rupture in adolescent boys. J Urol 184 (2010), p. 1733

5. Wang Z, Yang J, Huang Y, Wang L, Liu L, Wei Y. Diagnosis and management of testicular rupture after blunt scrotal trauma: a literature review. Int Urol Nephrol (2016) 48:1967-76

6. Buckley J. C., McAninch J. W. Use of ultrasonography for the diagnosis of testicular injuries in blunt scrotal trauma. J Urol 2006;175(1):175-178. doi: 10.1016/S00225347(05)00048-0.

7. Ferguson GG, Brandes SB. Gunshot wound injury of the testis: the use of tunica vaginalis and polytetrafluoroethylene grafts for reconstruction. $J$ Urol 2007;178(6):2462-5.

8. Kapoor D, Leech J, Yap W. Use of tunica vaginalis patch graft for repair of traumatic testicular rupture. Urology 1992; 40: 374-5.

9. Tahtali IN, Halis F, Yildiz T, Gokce A, Ilçe Z, Balabay MD. Comparison of Polypropylene Mesh and Primary Repair in the Treatment of Blunt Testicular Rupture. Urol J 2016 Oct 10;13(5):2864-2868.

10. Marchalik D, Triest JA, Wright HC, Bandi G. Use of "off the shelf" extracellular matrix graft materials for repair of testicular rupture: a novel technique. Urology 2014 Sep;84(3):719-21. doi: 10.1016/j.urology.2014.05.026. 
Figures and Tables

Fig. 1.

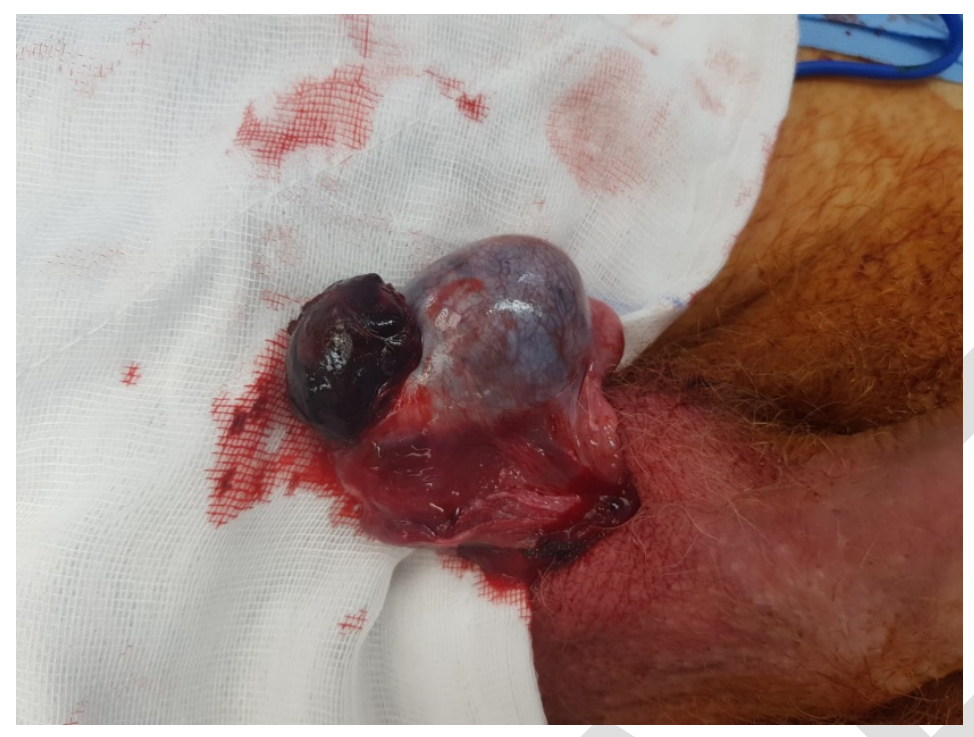

Fig. 2.

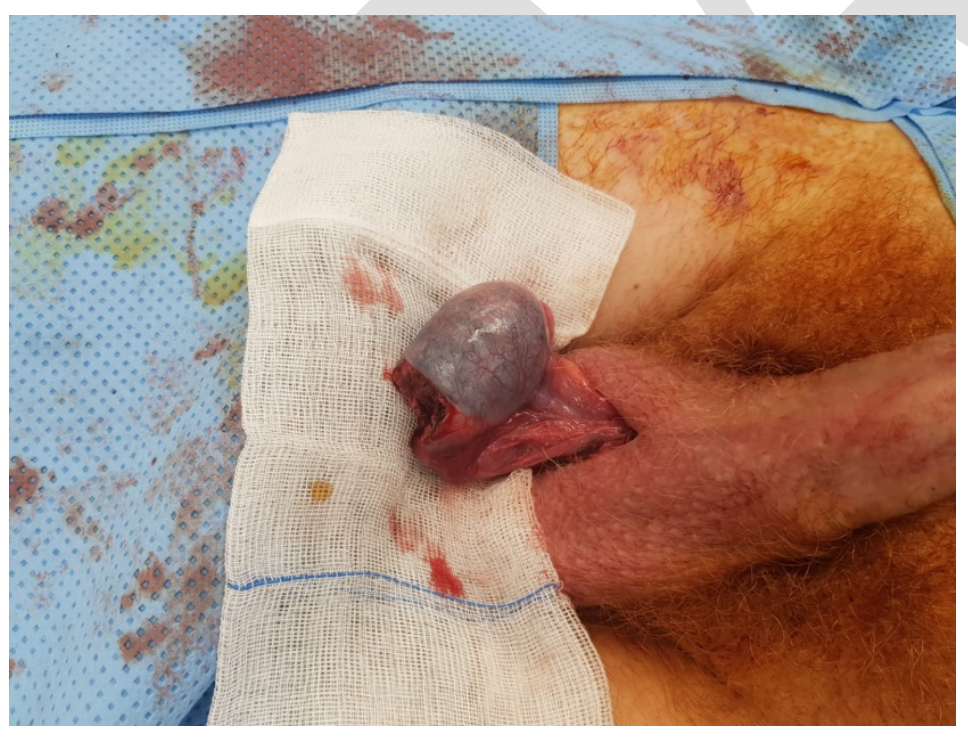


Fig. 3.

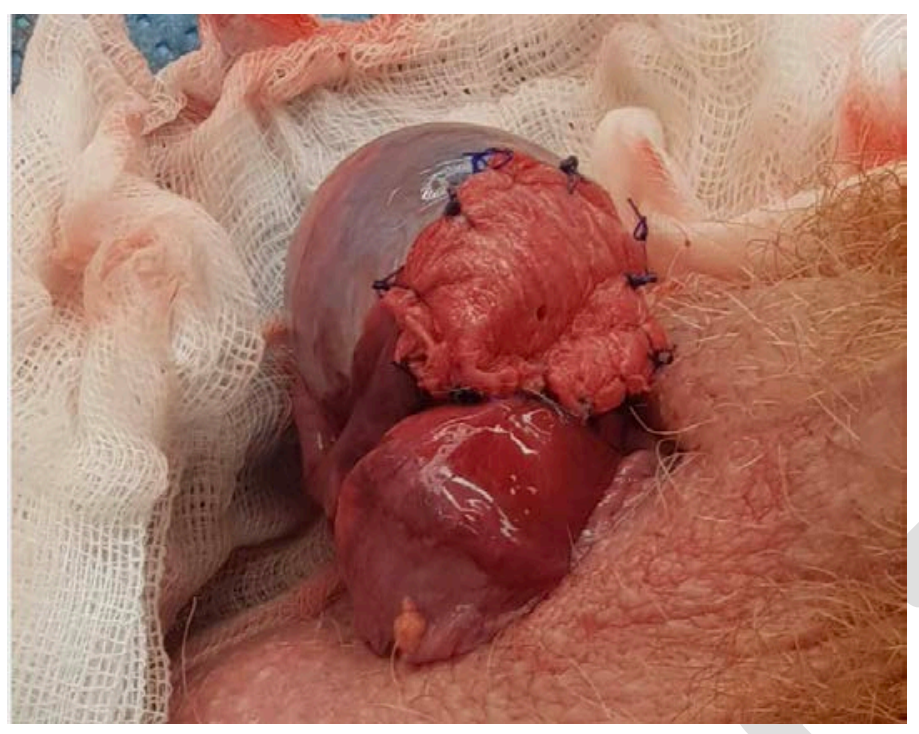

